\title{
Evaluation of Masticatory Muscles Function in Different Malocclusion Cases Using Surface Electromyography
}

\author{
Shamima Easmin Nishi ${ }^{1)}$, Rehana Basri ${ }^{2 *}$, Mohammad Khursheed Alam ${ }^{3)}$, Shinichi Komatsu ${ }^{4}$, Atsuo Komori ${ }^{4}$, \\ Yoshihiko Sugita ${ }^{4.5)}$ and Hatsuhiko Maeda ${ }^{4.5}$ \\ 1) Orthodontic Unit, School of Dental Science, Universiti Sains Malaysia, Kubang Kerian, Kota Bharu, Kelantan, Malaysia. \\ 2) Craniofacial Biology, School of Dental Science, Universiti Sains Malaysia, Kubang Kerian, Kota Bharu, Kelantan, Malaysia. \\ 3) Orthodontic Department, Collage of Dentistry, Al Jouf University,Sakaka, Kingdom of Saudi Arabia \\ 4) Department of Oral Pathology, School of Dentistry, Aichi Gakuin University, Nagoya, Japan \\ 5) Center of Advanced Oral Science, Aichi Gakuin University, Nagoya, Japan
}

(Accepted for publication, September 27, 2016)

\begin{abstract}
A malocclusion is a misalignment or incorrect relation between the teeth of the two dental arches when they approach each other as the jaws close. It has been suggested that masticatory function varies in relation to different malocclusion. Thereby, masticatory muscle can be affected with different malocclusion as masticatory process is related with this. The aim of this study was to evaluate the function of masticatory muscles (masseter and temporalis muscles) in patient with different malocclusion by surface electromyography (sEMG). On masticatory muscle, twenty eight patients (13 male and 15 female) were assessed by using sEMG and the results compared between gender and different malocclusions. Male and female group in left masseter muscle during chewing and right temporalis muscle during clenching. No significant difference $(\mathrm{p}>0.05)$ for class I, II and III malocclusion, except right temporalis muscle showed significant difference $(\mathrm{p}=0.010)$ on clenching. In conclusion, the function of the masticatory muscles were varies between male and female and different malocclusion of class I, II and III.
\end{abstract}

Key words: Malocclusions, Masticatory muscles, Electromyography

\section{Introduction}

The malocclusions are very common in modern generation which caused by misalignment or incorrect relation between the teeth of two dental arches when they approach each other as the jaws close ${ }^{1-2)}$. According to Edward Angle's classification of malocclusion, it can be 3 types: Class I, II and III. Angle's classification was based on the mesio-distal relation of the teeth, dental arches and the jaws ${ }^{3}$. The classification be determined by on where the buccal groove of the mandibular first molar contacts the mesiobuccal cusp of the maxillary first molar: on the cusp (Class I, neutroclusion, or normal occlusion); distal to the cusp by at least the width of a premolar (Class II, distocclusion); or mesial to the cusp (Class III, mesiocclusion) ${ }^{3}$.

Angle's class I malocclusion present with normal inter-arch molar relation. Patients with class I malocclusion exhibits normal skeletal relation with normal muscle function ${ }^{3)}$. Class II malocclusion is characterized when the disto-buccal cusp of the upper first permanent molar occludes in the buccal groove of the

Correspondence to: Dr. Rehana Basri, Craniofacial Biology, School of Dental Science, Universiti Sains Malaysia, Kubang Kerian 16150, Kota Bharu, Kelantan, Malaysia; Tel: 6097675888; Fax: 607642026; E-mail: drlamisha@gmail.com lower first permanent molar. Class II malocclusions are most frequent problem faced in orthodontic practice ${ }^{4}$. Angle has subclassified class II malocclusions into two divisions: class II division 1 and class II division 2. Class II division 1 malocclusion patient exhibits hyperactive buccinators and mentalis muscle function with altered tongue position ${ }^{3)}$. Class II division 2 patients exhibit normal perioral muscle activity ${ }^{5}$. Class III malocclusion characterized by a class III molar relation with the mesio-buccal cusp of the maxillary first permanent molar occluding in the interdental space between the mandibular $1^{\text {st }}$ and $2^{\text {nd }}$ molars ${ }^{3)}$. It can be true class III and Pseudo class III. Class III malocclusion appears with altered muscle function ${ }^{5}$.

Mastication procedure involves chewing, swallowing and digestion of food where the occlusal surface of post-canine teeth is involved for crushing of solid foods. In this process, masticatory movements are associated with the masseter, temporalis, medial and lateral pterygoid muscles, together with the submental muscles, i.e. the digastric, mylohyoid and geniohyoid muscles ${ }^{6}$. Neuromuscular disorder negatively influence oral functions and orofacial morphology and these diseased muscles causes changing of malocclusions such as anterior and posterior open bites or cross 
bite etc. $^{7-8)}$.

Electromyography is the recording of the electrical activity of muscle tissue, or its representation as a visual display or audible signal, using electrodes attached to the skin or inserted into the muscle $^{9}$. Surface electromyography is mainly performed by an instrument called electromyography and that produce a record called an electromyogram ${ }^{10)}$. sEMG used to assess muscle function by recording muscle activity from the surface above muscle on the skin using a pair of electrodes. Because EMG recording shows the potential difference between two separate electrodes. Surface electromyography permits the non-invasive investigation of the bioelectrical phenomena of muscular contraction ${ }^{11)}$. sEMG adequately allows the examination of some important muscles involved in chewing, swallowing and posture of the head (typically masseter, temporalis anterior and posterior, digastric anterior, sternocleidomastoid) ${ }^{12}$. Usually, the masseter and the temporalis are synergists and function concurrently. Whereas the temporalis provides a basis for mandibular balance and postural control, the masseter is responsible for grinding and chewing ${ }^{13)}$. Malocclusion can negatively affect the masticatory process (like chewing, clenching) where masticatory muscle also get affected. It has been suggested that masticatory function varies in relation to different malocclusion (class I, II and III) ${ }^{12}$.

The aim of the present study was to assess the function of masticatory muscles (the masseter and temporalis muscles) in patients with different gender and malocclusion (class I, II, III) by surface electromyography (sEMG).

\section{Materials and Methods}

In this study, all participants provide their verbal and written informed consent. This study was approved by the Ethical Committee of the Hospital Universiti Sains Malaysia (HUSM). Letter from Human Research Ethics Committee (HREC) has assigned a study protocol code USM/ JEPeM/1405200.

Subjects and Sample Selection

The subjects of this study consisted of a total of 28 subjects (13 male and 15 female), age group was 18 to 25 years of age. Subject group were refer to patients of School of Dental Science, Hospital University Sains Malaysia (HUSM).

\section{Inclusion criteria}

1. The patients with different malocclusion (Class I, II and III).

2. Age between 18 to 26 years.

3. Patients with all sound erupted permanent teeth (except considering $3^{\text {rd }}$ molars).

\section{Exclusion criteria}

1. Patients with congenital / craniofacial deformities.

2. Unhealthy patients (patients with TMJ dysfunction, parafunctional habit etc).
3. Patients having pathology within the maxilla or mandible.

\section{Clinical examination}

Intraoral examination was done to detect different malocclusion according to Angle's classification of malocclusion ${ }^{3}$.

\section{Electromyography}

After the clinical examination, surface electromyography (sEMG) of the masseter and temporalis muscles was done using an electromyography device (ProComp Infiniti Encoder SA7500, Montreal, Quebec, Canada). There were total 3 surface leads, 2 electrods were recording electrode and another one was reference electrode for each side to record muscle activity for the masseter and temporalis muscle respectively. For temporalis muscle a line were drawn from upper Earline to canthus of the eye and the electrode were placed above this line by palpating muscle contraction position. In case of masseter muscle, after palpating angle of mandible, the electrode was placed over muscle (anterosuperior to the angle of the mandible). The patients were given clear instructions before the sEMG recording started. The instructions were:

- To keep the head and body still as movement of the head and neck region or the body might affect the sEMG results.

- Do not move the tongue as it would result in stimulation to the muscles thus affecting the results.

- A chewing gum was given, but chewing were only started on signal provided.

- The surface leads were placed at different areas respectively: started with left masseter, then right masseter, followed by left temporal region and ended with right temporal region.

- The sEMG recording was started with the left masseter after surface lead placement with the patients' masticatory musclesbeing at rest for 10 seconds, followed by 10 seconds of chewing on the left side, then 10 seconds of rest and after that 10 seconds of continuous clenching, ending with another 10 seconds of rest. - Next, the surface leads were placed on the right masseter region for sEMG recording with the same instructions.

- For the left and right temporalis muscle, patients were instructed to rest for 10 seconds, followed by continuous clenching for 10 seconds and ending with another 10 seconds of rest.

\section{Statistical analysis}

The data were verified and analyzed statistically using IBM SPSS Statistics Version 22.0 (Armonk, NY: IBM Corp.) with confidence level set at $5 \%(\mathrm{P}<0.05)$ to test for significance. Mean amplitude of the muscles (masseter and temporalis) between male and female patients were analyzed by $t$ test. Mean amplitude of the muscles (masseter and temporalis) in patients with class I, II and III during basal stage (resting), post chew resting stage, post 


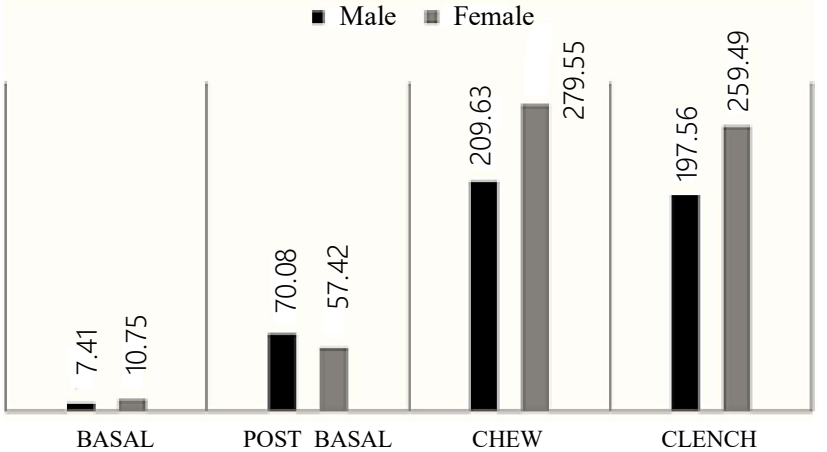

Figure 1. Mean amplitude of the right masseter between male and female patient

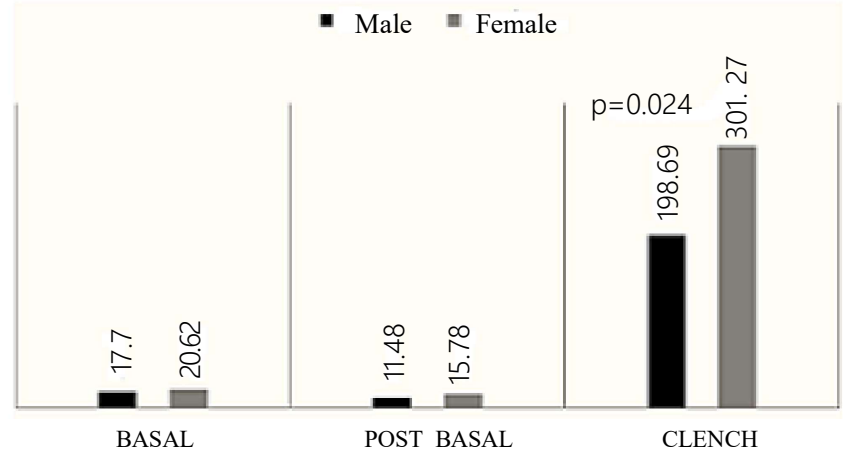

Figure 3. Mean amplitude of the right temporalis between male and female patient

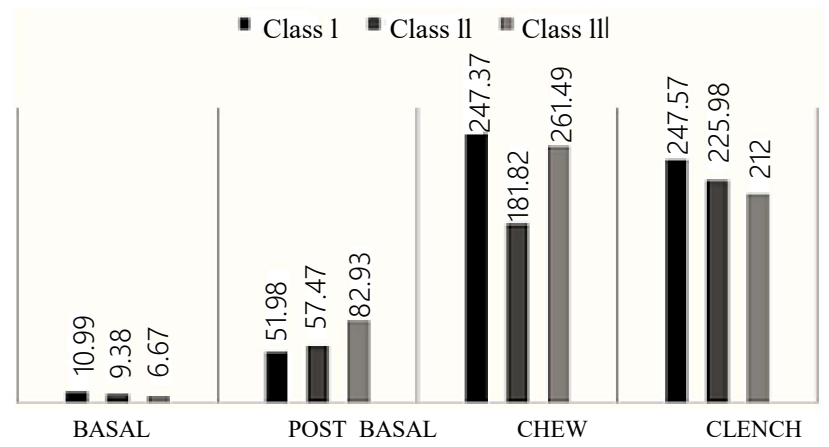

Figure 5. Mean amplitude of the right masseter muscle activity among class I, II and III malocclusion groups

clenching basal stage (resting), left chewing stage, right chewing stage and during clenching stage was analyzed by One-Way ANOVA.

\section{Result}

\section{Gender disparities}

In right masseter muscle, mean value was higher in female group than the male (Fig. 1). But only post basal mean value showed higher in male $(57.42 \mathrm{mV})$ than female $(70.09 \mathrm{mV})$. Here, right masseter muscle showed no significant difference $(p>0.05)$ for male and female groups. Left masseter muscle showed

- Male E Female

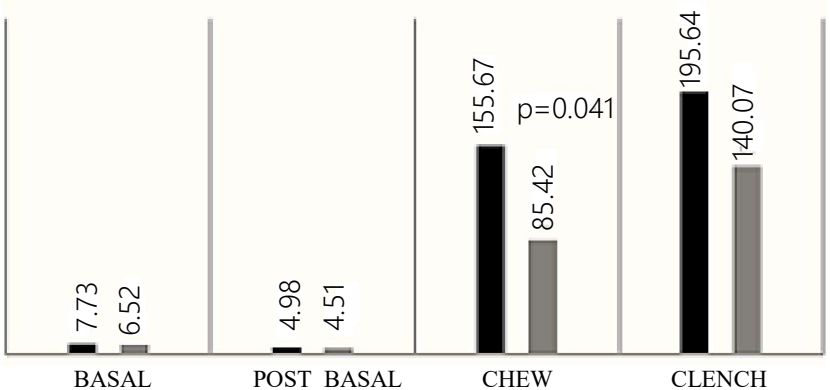

Figure 2. Mean amplitude of the left masseter between male and female patient

- Male $\square$ Female

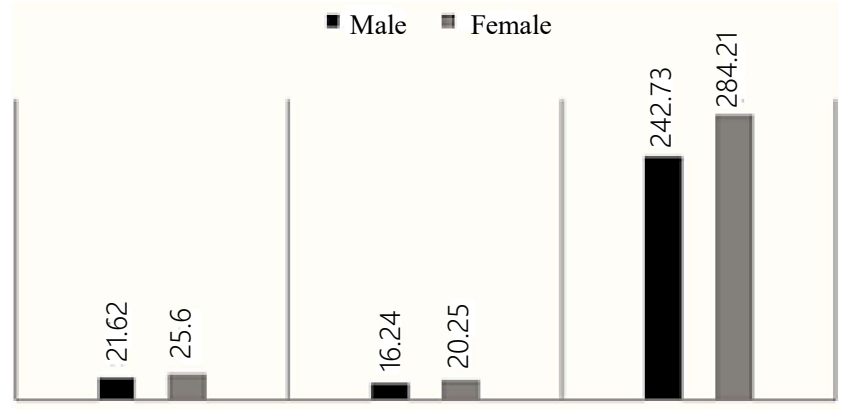

BASAL

POST BASAL

CLENCH

Figure 4. Mean amplitude of the left temporalis between male and female patient

- Class 1 a Class 11 " Class 111

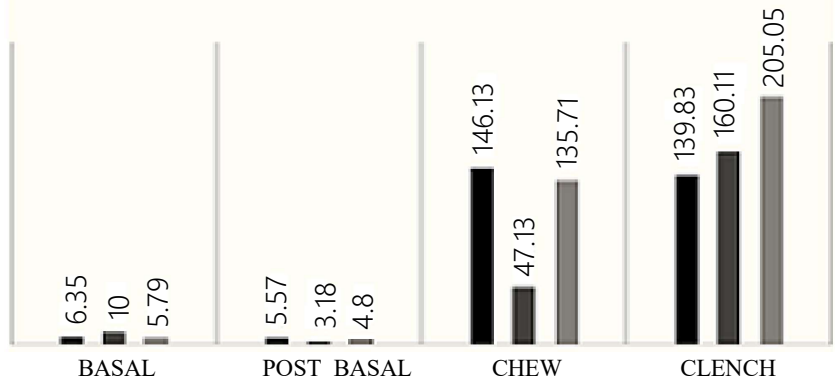

Figure 6. Mean amplitude of the left masseter muscle activity among class I, II and III malocclusion groups

significantly different $(\mathrm{p}=0.041)$ for male and female group during chewing (Fig .2). Here mean value of male patient was $155.67 \mathrm{mV}$ and for female patient was $85.42 \mathrm{mV}$. Right temporalis muscle also showed significantly different $(\mathrm{p}=0.024)$ during clenching (Fig. 3). Where the mean value was $198.69 \mathrm{mV}$ for male patient and $301.27 \mathrm{mV}$ for female patient. Left temporalis muscle showed no significant difference for male and female group during basal stage (resting), post basal (resting after clenching) and clenching (Fig. 4).

\section{Malocclusion disparities}


- Class 1 e Class 11 e Class 111

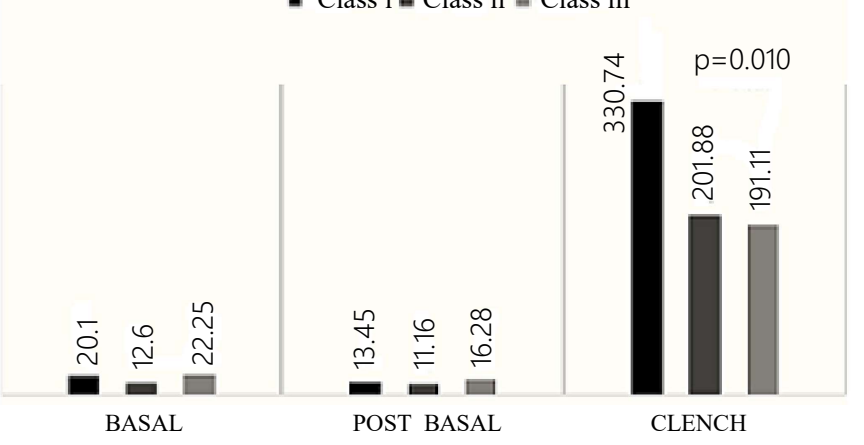

Figure 7. Mean amplitude of the right temporalis muscle activity among class I, II and III malocclusion groups

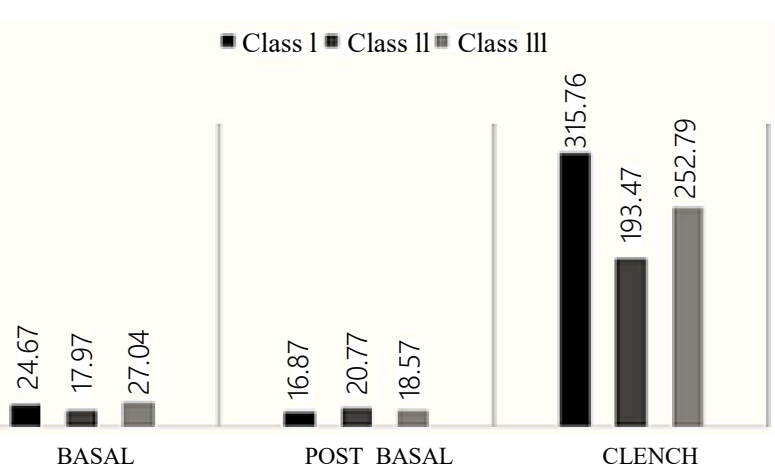

Figure 8. Mean amplitude of the left temporalis muscle activity among class I, II and III malocclusion groups

Table 1. Comparison of present study with different study

\begin{tabular}{|c|c|c|c|c|c|}
\hline Year & Author & Muscle & Action & Malocclusion & Result \\
\hline 1980 & $\begin{array}{l}\text { Pancherz and } \\
\text { Anehus }^{17)}\end{array}$ & $\begin{array}{l}\text { Masseter and } \\
\text { temporalis }\end{array}$ & $\begin{array}{l}\text { Chewing and } \\
\text { Clenching }\end{array}$ & $\begin{array}{l}\text { Class II division } 1 \\
\text { and normal occlusion }\end{array}$ & $\begin{array}{l}\text { Masseter muscle during } \\
\text { chewing less EMG activity } \\
\text { in boys with class II } \\
\text { division } 1 .\end{array}$ \\
\hline 1986 & Ahlgren ${ }^{23)}$ & Temporalis & Clenching & Normal occlusion & No significant difference. \\
\hline 1988 & Miralles et. $a l^{18)}$ & $\begin{array}{l}\text { Masseter and } \\
\text { temporalis }\end{array}$ & $\begin{array}{l}\text { Maximal } \\
\text { voluntary } \\
\text { clenching } \\
\text { (MVC) }\end{array}$ & Class II division 1 & No significant difference. \\
\hline 2012 & Piancino et.al ${ }^{24)}$ & $\begin{array}{l}\text { Masseter and } \\
\text { temporalis }\end{array}$ & Chewing & $\begin{array}{l}\text { Class I malocclusion } \\
\text { with open bite and } \\
\text { normal occlusion }\end{array}$ & Significantly different. \\
\hline 2013 & $\mathrm{KO}$ et. $\mathrm{al}^{19)}$ & $\begin{array}{l}\text { Masseter and } \\
\text { temporalis }\end{array}$ & $\begin{array}{l}\text { Maximal } \\
\text { voluntary } \\
\text { clench }\end{array}$ & $\begin{array}{l}\text { Class III (before and } \\
\text { after treatment) }\end{array}$ & No significant difference. \\
\hline 2016 & Piancino et.al $l^{20)}$ & Masseter & Chewing & $\begin{array}{l}\text { Unilateral posterior } \\
\text { cross bite and normal } \\
\text { occlusion }\end{array}$ & Significantly different. \\
\hline 2016 & Present study & $\begin{array}{l}\text { Masseter and } \\
\text { temporalis }\end{array}$ & $\begin{array}{l}\text { Chewing and } \\
\text { clenching }\end{array}$ & $\begin{array}{l}\text { Class I,II and III } \\
\text { malocclusion }\end{array}$ & Significantly different. \\
\hline
\end{tabular}

Using ANOVA (Bonferroni) right and left masseter muscle showed no significant difference for basal, post basal, chewing and clenching in class I, II and III malocclusion respectively (Fig $5 \& 6$ ). Only right temporalis muscle showed significant difference $(p=0.010)$ on clench (Fig 7). Where mean value was $330.74 \mathrm{mV}$ in class I malocclusion, $201.88 \mathrm{mV}$ in Class II and $191.11 \mathrm{mV}$ in class III malocclusion. Left temporalis muscle showed no significant difference for basal, post basal and clenching in class I, II and III malocclusion respectively (Fig 8).

\section{Discussion}

Different malocclusion (class I, II and III) can alter muscle activity which can act as a predisposing factor for more sever malocclusion. This study have been evaluated the functional ability of masticatory muscle in different malocclusion by using surface electromyography (sEMG). Patient with different malocclusion may show different efficacy of masticatory muscle. Masticatory muscle with neuromuscular disorder can act as an aggravating factor for malocclusion. For different malocclusion patient may adapt faulty masticatory positon which may cause interference to orthodontic treatment.

Present study compared the muscle activity of masseter and temporalis in male and female group during basal or rest, post basal, chewing and clench. In current study left masseter muscle was more active during chewing in male patient than female. On the other hand right temporalis muscle was more active in female patient during clenching. Craniofacial structure for male and female varies in diameter and length. Masticatory muscle activity has been determined to be associated with craniofacial morphology ${ }^{14)}$. Therefore, muscular arrangement can act as a fact for this active EMG response in male and female group. Masseter and temporalis are important masticatory muscle as they act by elevation, proclination and retraction of mandible. Thus the chewing and clenching force can be affect by misaligned teeth ${ }^{12}$. 
Shamima Easmin Nishi et al.: Muscle Ability in Different Malocclusion

The relationship between EMG and craniofacial morphology, were identified high EMG activity of the masseter muscle in children with normal occlusion ${ }^{14)}$. It has been reported that the onset of temporal muscle activity was involved in the positioning of the mandible during masticatory movement in patients with normal occlusion and that the temporal muscle was activated earlier than the masseter muscle ${ }^{15)}$ (Table 1).

Moyer $^{16)}$ using surface electrode and unipolar recording, where result found that balanced activity in all three parts of the temporalis muscle (anterior, middle, and posterior) at rest and during habitual clenching in normal occlusion. In Angle Class II, Division 1 there were uneven EMG activity with a strong dominance of the posterior regions. In present study, right temporalis muscle showed more activity during clenching in male patient than female.

Pancherz et al. ${ }^{17)}$ conducted a study to evaluate the effect of continuous bite jumping in masticatory muscles in ten growing boys with Class II, Division I malocclusion cases treated with the Herbst appliance. They analyzed EMG from the temporal and masseter muscles during maximal biting in intercuspal position and during chewing of peanuts. They found that the EMG activity from the temporal and masseter muscles was reduced in Class II, Division 1 malocclusions when compared to normal occlusions. But a significant increase in EMG activity was found, especially for the masseter muscles during maximal biting. In this study, right temporalis muscle of different malocclusion group showed more response for class I malocclusion during clenching than class II and class III. Right and left masseter muscle showed no significant difference during chewing and clenching in class I, II and III malocclusion.

According to a study by Miralles et al. ${ }^{18)}$ that aims to evaluate EMG activities of masseter and anterior temporalis muscle during maximal voluntary clenching in class II division 1 patient and the result showed no significant difference. In present study also suggested class II malocclusion patient showed no significant difference during clenching in masseter and temporalis muscle. Ko et al. ${ }^{19)}$ Class III patient before orthognathic surgery showed more EMG response then after surgery. In current study, Class I patient showed high EMG activity than class II and class III. The effects of therapy on masseter activity and chewing kinematic in patients having unilateral posterior cross bite was undergone for a study introduced by Piancino et al. ${ }^{20)}$ in 2016 . They analyzed on the mandibular motion and the muscular activity during chewing soft and hard boli were consecutively recorded, before and after correction with function producing bite. There were significant reduction of masseter muscles activity with reverse chewing patterns. In current study masseter activity had been increased during normal chewing activity for male patient. Multi-channel EMG recordings are indicated to compare activation patterns between several muscles or muscle regions and it can provide data for individual muscle activity at the same time ${ }^{21)}$. Hence, recording of chewing activity in several muscle in same time, may avoid electrical error with more accurate data ${ }^{21)}$. sEMG result provides electrical activity in muscles and numerous factors like rate of muscle stimulation, size and morphology of motor units, electrical properties of the tissues can alter electrical signal of $\mathrm{EMG}^{22)}$.

There are various method of EMG recording. Mainly it depends on muscle function or expression while recording. However, the present study reveals the masticatory muscle function using sEMG. This study has not focused on the methods of EMG and had not compared with the EMG methods with other reports. Therefore, it can be a topics of interest in future research and need to pay more attention on this. As, EMG is a clinical approach and requires careful handling for accurate recording. In consequence it will be beneficial for clinicians during EMG recording.

In conclusion, this study showed right temporalis muscle activities were significantly high in male group and different malocclusion group. Right masseter muscle activities showed no significant difference in gender and different malocclusion groups. Left masseter muscle showed increased chewing activities in male group than female group. Patient with class I malocclusion group found higher EMG activity than class II and class III malocclusion group.

\section{Acknowledgement}

USM short-term grant (304/PPSG/61312134), USM RU grant 1001/PPSG/812154 and USM fellowship.

\section{Conflict of Interest}

The authors have declared that no conflict of interest.

\section{Reference}

1. Sarver DM, Proffit WR and Ackerman JL. Diagnosis and treatment planning in orthodontics. In: Graber TM, Vanarsdall RL Jr, eds. Orthodontics, Current Principles and Techniques. St Louis: Mosby, 3: 3-15, 2000

2. Bishara SE. Textbook of Orthodontics, An approach to the diagnosis of different malocclusion. 13: 146-184, 2001

3. Iyyer B, Bhalajhi SI and Bhalajhi SI. Orthodontics: The art and science. Arya (Medi) Publ, 2012

4. Suresh R and Priya K. Orthodontic camouflage treatment of skeletal class II malocclusion with severe maxillary dentoalveolar protrusion. J Pierre Fauchard Acad 27: 118123, 2013

5. Singh G. Textbook of Orthodontics. JP Medical Ltd, City, 2015

6. Le Révérend BJ, Edelson LR and Loret C. Anatomical, functional, physiological and behavioural aspects of the 
development of mastication in early childhood. Br J Nutr 111: 403-414, 2014

7. Katsaros SKC. The effects of myotonic dystrophy and Duchenne muscular dystrophy on the orofacial muscles and dentofacial morphology. Acta Odontol Scand 56: 369-374, 1998

8. van Bruggen HW, van de Engel-Hoek L, Steenks MH, Bronkhorst EM, Creugers NHJ, de Groot IJM and Kalaykova SI. Predictive factors for masticatory performance in Duchenne muscular dystrophy. Neuromuscul Disord 24: 684-692, 2014

9. McQuillen MP. Clinical electromyography: definition, application, and innovation. Adv Neurol 17: 285-294, 1976

10. Alam MK, Rahman SA, Basri R, Yi TTS, Si-Jie JW and Saha S. Correction: Dental Implants-Perceiving Patients' Satisfaction in Relation to Clinical and Electromyography Study on Implant Patients. PloS one 10 (11): e0140438, 2015

11. Ferrario VF, Sforza C, D'addona A and Barbini E. Electromyographic activity of human masticatory muscles in normal young people. Statistical evaluation of reference values for clinical applications. J Oral Rehabil 20: 271-280, 1993

12. Iyer $\mathrm{M}$ and Valiathan $\mathrm{A}$. Electromyography and its application in orthodontics. Curr Sci (Bangalore) 80: 503-6, 2001

13. Criswell E. Cram's introduction to surface electromyography. Jones \& Bartlett Publishers, City, 2010

14. Nakamura K, Hara A, Nakata S, Hyakutake H and Takahashi I. Relationship between the stability of muscle activity in the masseter muscle and craniofacial morphology. Orthod Waves 72: 55-62, 2013

15. Perry HT and Harris SC. Role of the neuromuscular system in functional activity of the mandible. J Am Dent Assoc 48: 665,1954
16. Moyers RE. Temporomandibular muscle contraction patterns in Angle Class II, Division 1 malocclusions: An electromyographic analysis. Am J Orthod 35(11): 837-857, 1949

17. Pancherz $\mathrm{H}$ and Anehus-Pancherz M. Muscle activity in Class II, Division malocclusions treated by bite jumping with the Herbst appliance: An electromyographic study. Am J Orthod 78: 321-329, 1980

18. Miralles R, Berger B, Bull R, Manns A, and Carvajal R.Influence of the activator on electromyographic activity of mandibular elevator muscles. Am J Orthod Dentofac Orthop 94: 97-103, 1988

19. Ko EWC, Teng TTY, Huang CS and Chen YR. The effect of early physiotherapy on the recovery of mandibular function after orthognathic surgery for class III correction. Part II: Electromyographic activity of masticatory muscles. J Craniomaxillofac Surg 43: 138-143, 2015

20. Piancino MG, Falla D, Merlo A, Vallelonga T, de Biase C, Dalessandri D and Cesare Debernardi. Effects of therapy on masseter activity and chewing kinematics in patients with unilateral posterior crossbite. Arch Oral Biol 67: 61-67, 2016

21. Schumann NP, Bongers K, Guntinas-Lichius O and Scholle HC. Facial muscle activation patterns in healthy male humans: A multi-channel surface EMG study. J Neurosci Methods 187: 120-128, 2010

22. Alam MK, Basri R and Reza MF. Myofunctional benefit of tooth brushing with Miswak Electromyography and Magnetoencephalography assessment. Int Med J 20: 247249, 2013

23. Ahlgren J. EMG pattern of temporalis in normal occlusion. Eur J Orthodont 8: 185-191, 1986

24. Piancino MG, Isola G, Merlo A, Dalessandri D, Debernardi $\mathrm{C}$ and Bracco P. Chewing pattern and muscular activation in open bite patients. J Electromyogr Kinesiol 22: 273-279, 2012 